\title{
REMARKS ON DEFECTIVE FANO MANIFOLDS
}

\author{
PALTIN IONESCU AND FRANCESCO RUSSO
}

Dedicated to the memory of Alexandru Lascu

\begin{abstract}
This note continues our previous work on special secant defective (specifically, conic connected and local quadratic entry locus) and dual defective manifolds. These are now well understood, except for the prime Fano ones. Here we add a few remarks on this case, completing the results in our papers [24], [11], [12], [13] and [14]; see also the recent book [25].
\end{abstract}

\section{INTRODUCTION}

Let $X \subset \mathbb{P}_{\mathbb{C}}^{N}:=\mathbb{P}^{N}$ be a closed subvariety, that we always assume to be smooth, irreducible, non-degenerate, of dimension $n$ and codimension c. The expected dimension of $S X$, the secant variety of $X$, is $2 n+1$. The difference between the expected and the actual dimension is the number $\delta \geqslant 0$, called the secant defect. If $\delta>0, X$ is said to be secant defective; this is the same as being (possibly after an isomorphic projection) of small codimension, in the sense that $n \geqslant c$. At present we understand better only some subclasses of secant defective manifolds: conic connected (CC for short) and local quadratic entry locus (LQEL for short) ones. CC manifolds are defined by the presence of a conic contained in $X$ and passing through two given general points. An important particular case of CC manifolds are the LQEL ones, defined by the presence of a quadric of dimension $\delta$, contained in $X$ and passing through two given general points; they were introduced in [24] and further studied in [11].

An embedded Fano manifold is prime if its Picard group is generated by the hyperplane section class, $H \in \operatorname{Pic}(X)$. In this case, its index $i$ is defined by the relation $-K_{X}=i H$ in $\operatorname{Pic}(X), K_{X}$ being the canonical class. The CC manifolds are Fano and classified, but for the case they are prime [12].

In sections 3 and 4, we investigate the property of being CC or LQEL for a Fano manifold of high index. For instance, we show that for Fano manifolds of index $i$ with $\delta \geqslant 2$ and $S X=\mathbb{P}^{N}$, "high index" implies "small

2010 Mathematics Subject Classification. 14MXX, 14NXX, $14 \mathrm{~J} 45$.

Key words and phrases. Fano manifold, conic connected, local quadratic entry locus, dual defective. 
codimension", see Corollary 3.9 (i). We characterize LQEL manifolds with $\delta \geqslant 3$ by the equality $i=\frac{n+\delta}{2}$, see Proposition 4.1. In Proposition 4.2 we obtain a classification result for Fano manifolds of high index and small codimension, based on [24].

If $X \subset \mathbb{P}^{N}$ is as before, it is called dual defective (DD for short) if its dual variety $X^{*} \subset \mathbb{P}^{N^{*}}$ is not a hypersurface. The number $k=N-1-\operatorname{dim}\left(X^{*}\right)$ is the dual defect of $X$. In section 5 we give ample evidence for our belief that prime Fano DD manifolds are LQEL, improving on [14]. In particular, we show that a DD manifold is LQEL if (and only if) the relation $\delta=k+2$ holds, Proposition 5.2.

In the last section we make some remarks about the case of quadratic manifolds (that includes all known examples of both LQEL and DD prime Fano manifolds in their natural embedding). We also mention the link with the famous Hartshorne Conjecture on manifolds of small codimension, that was shown to hold in the quadratic case [13].

We thank the anonymous referee for her/his suggestions that improved both the exposition and point (v) of our Proposition 5.3.

\section{PRELIMINARIES}

We work over $\mathbb{C}$ and use the customary notation in algebraic geometry, as in [14]; in particular, $\mathbb{P}(E)$ is intended in Grothendieck's sense. We denote by $X \subset \mathbb{P}^{N}$ a smooth, irreducible, non-degenerate embedded projective variety of dimension $n$ and codimension $c$. For a point $x \in X$, we write $\mathbf{T}_{x} X$ for the Zariski tangent space at $x$ and $T_{x} X$ for its projective closure in $\mathbb{P}^{N}$. $\mathcal{P}_{X}$ denotes the first jet bundle of $\mathcal{O}_{X}(1)$. If $N_{X / \mathbb{P}^{N}}$ is the normal bundle of $X$ in $\mathbb{P}^{N}$, we have the standard exact sequence:

$$
0 \rightarrow N_{X / \mathbb{P}^{N}}^{*}(1) \rightarrow \mathcal{O}_{X}^{N+1} \rightarrow \mathcal{P}_{X} \rightarrow 0
$$

Definition 2.1. $X$ is Fano if $-K_{X}$ is ample. $X \subset \mathbb{P}^{N}$ is called prime Fano if it is Fano and $\operatorname{Pic}(X)=\mathbb{Z}[H]$, where $H$ denotes the hyperplane section class; it is called Fano of index $i>0$ if $-K_{X}=i H$.

Remark 2.2. Note that our definition of the index of an embedded Fano manifold is different from the usual one, but is the same if $X$ is prime.

Lemma 2.3. (i) Let $X$ be a Fano manifold (of dimension $n$ ) and assume that $-K_{X}=j D$, for some ample divisor $D$. Then $j \leqslant n+1$.

(ii) Let $X \subset \mathbb{P}^{N}$ be a Fano manifold of index $i \geqslant \frac{n+3}{2}$. Then $X$ is prime Fano.

Proof. (i) follows from vanishing results, see [16]. 
(ii) A result by Wiśniewski [26] shows that $\operatorname{Pic}(X)$ is cyclic. By (i), $H$ cannot be divisible, so $X$ is prime.

Prime Fano manifolds of high index, other than complete intersections, are quite special.

Definition 2.4. $X \subset \mathbb{P}^{N}$ is covered by lines if for any point $x \in X$, there is a line $l \subset X$ such that $x \in l$.

The following fundamental fact, coming from [19], shows that Fano manifolds of high index are covered by lines.

Lemma 2.5. If $X \subset \mathbb{P}^{N}$ is Fano of index $i$ and $i \geqslant \frac{n+2}{2}$, then $X$ is covered by lines.

If $X \subset \mathbb{P}^{N}$ is covered by lines and $x \in X$ is a general point, we denote by $\mathcal{L}_{x} \subset \mathbb{P}\left(\mathbf{T}_{x}^{*} X\right)$ the Hilbert scheme of lines contained in $X$ and passing through $x . \mathcal{L}_{x}$ is smooth and we let $a:=\operatorname{dim}\left(\mathcal{L}_{x}\right)$. If $X \subset \mathbb{P}^{N}$ is Fano of index $i$, we have $i=a+2$ and $\mathcal{L}_{x}$ is equidimensional.

Definition 2.6. The secant variety of $X \subset \mathbb{P}^{N}$, denoted by $S X$, is the closure of the locus of its secant lines. We have that $\operatorname{dim}(S X) \leqslant 2 n+1$ and $\delta:=2 n+1-\operatorname{dim}(S X)$ is the secant defect of $X . X$ is secant defective if $\delta>0$. The tangent variety of $X$, denoted by $T X$, is $T X=\bigcup_{x \in X} T_{x} X$. Let us also recall that, if $p \in S X$ is a general point, the entry locus of $X$ with respect to $p$, denoted by $\Sigma_{p}(X)$, is the intersection of $X$ with the cone of secants to $X$ passing through $p$. The secant defect of $X$ is $\operatorname{dim}\left(\Sigma_{p}(X)\right)$, for $p \in S X$ a general point.

Lemma 2.7. Let $X \subset \mathbb{P}^{N}$ be prime Fano of index $i \geqslant \frac{n+2}{2}$ and let $x \in X$ be a general point. Then one of the following holds:

(i) $S \mathcal{L}_{x}=\mathbb{P}^{n-1}$, or

(ii) $N_{\mathcal{L}_{x} / \mathbb{P}^{n-1}}(-1)$ is not ample.

Proof. If $X \subset \mathbb{P}^{N}$ we have that $\delta \geqslant n-c+1$ and equality holds if and only if $S X=\mathbb{P}^{N}$. Apply this to $\mathcal{L}_{x} \subset \mathbb{P}^{n-1}$ (note that $\mathcal{L}_{x} \subset \mathbb{P}^{n-1}$ is nondegenerate by [9, Thm. 2.5]). Now, assume that (i) does not hold. It follows that $\delta\left(\mathcal{L}_{x}\right)>a-(n-1-a)+1 \geqslant 0$. By Terracini's Lemma, see for instance [25. Thm. 1.4.1], there is a hyperplane in $\mathbb{P}^{n-1}$ such that its tangency locus contains the general entry locus $\Sigma_{p}\left(\mathcal{L}_{x}\right)$. As $\operatorname{dim}\left(\Sigma_{p}\left(\mathcal{L}_{x}\right)\right)=\delta\left(\mathcal{L}_{x}\right)>0$, (ii) follows.

Definition 2.8 ([24]). $X \subset \mathbb{P}^{N}$ is a local quadratic entry locus variety, abbreviated LQEL, if, given two general points $x, x^{\prime} \in X$, there is a quadric of dimension $\delta>0$ contained in $X$ and containing the points $x, x^{\prime}$. 
Note that any quadric $Q \subset X$ passing through the general points $x, x^{\prime} \in$ $X$ is part of the entry locus of $X$ with respect to a general point $p$ of the line $\left\langle x, x^{\prime}\right\rangle$. So the dimension of $Q$ is at most the secant defect of $X$. Thus, LQEL varieties are characterized by the fact that the general entry locus is a (finite) union of quadrics. LQEL varieties are the simplest secant defective manifolds; they were introduced in [24] and further studied in [11] and [12].

Definition 2.9. $X \subset \mathbb{P}^{N}$ is conic connected, abbreviated CC, if, given two general points $x, x^{\prime} \in X$, there is a conic $C \subset X$ such that $x, x^{\prime} \in C . X$ is connected by degenerate conics if $C$ is a pair of incident lines.

Obviously, LQEL manifolds are CC, and CC manifolds are secant defective. CC manifolds were studied in [12], where a complete classification was obtained, except for the case of prime Fano manifolds. In particular, the same applies for LQEL manifolds.

Definition 2.10. $X \subset \mathbb{P}^{N}$ is dual defective, of dual defect $k$, if $\operatorname{dim}\left(X^{*}\right)=$ $N-1-k$, where $X^{*} \subset \mathbb{P}^{N^{*}}$ is the dual of $X$ and $k>0$.

In [3] the classification of DD manifolds was reduced to that of prime Fano ones.

Let $X$ be a Fano manifold of dimension $n$ with $-K_{X}=i H, i>0$, for some ample divisor $H$; let $E$ be a spanned vector bundle on $X$, of rank $r$. Let $Y:=\mathbb{P}(E)$, let $M:=\mathcal{O}_{Y}(1)$ and assume that $M^{n+r-1}=0$. Let $\varphi: Y \rightarrow W$ be the Stein factorization of the map associated to $|M|$ and let $F$ be its general fiber. Finally, assume that $\operatorname{det}(E)=j H$.

Proposition 2.11. Under these assumptions, $F$ is Fano if and only if $j<i$. If this is true and $\operatorname{Pic}(X)$ is cyclic, then $Y$ is also Fano and $\varphi$ is its second Mori contraction.

Proof. We have $-K_{Y}=r M-\pi^{*}\left(K_{X}+\operatorname{det}(E)\right)$, where $\pi: Y \rightarrow X$ is the projection. Restricting to $F$ gives: $-K_{F}=-\left.\left(K_{X}+\operatorname{det}(E)\right)\right|_{F}=$ $(i-j) H_{F}$. The rest is clear, since $\operatorname{Pic}(Y)=\mathbb{Z} \oplus \mathbb{Z}$ and the cone of curves of $Y$ is generated by two classes: one of a curve contracted by $\pi$ and one of a curve contracted by $\varphi$.

Corollary 2.12. Let $X \subset \mathbb{P}^{N}$ be Fano of index $i$.

(i) Assume $\delta \geqslant 2$ and let $E=\mathcal{P}_{X}$. Then $F$ is Fano if and only if $i \geqslant \frac{n+2}{2}$. In particular $i \geqslant \frac{n+2}{2}$ implies $i \leqslant \frac{n+\delta+1}{2}$ with equality holding if and only if $F \simeq \mathbb{P}^{\delta-1}$.

(ii) If $\delta \geqslant 2$ and $X$ is prime, then $\varphi: \mathbb{P}\left(\mathcal{P}_{X}\right) \rightarrow W$ is a Mori contraction if and only if $i \geqslant \frac{n+2}{2}$. 
(iii) Assume that $k>0$ and let $E:=N_{X / \mathbb{P}^{N}}(-1)$. Then $\varphi: \mathbb{P}(E) \rightarrow W$ is a Mori contraction, $i=\frac{n+k+2}{2}$ and $X$ is prime.

Proof. (i) From (1) we have the natural map $\varphi^{\prime}: \mathbb{P}\left(\mathcal{P}_{X}\right) \rightarrow T X \subset \mathbb{P}^{N}$, whose Stein factorization is $\varphi: \mathbb{P}\left(\mathcal{P}_{X}\right) \rightarrow W$. Since $\delta>0$, we have $\operatorname{dim}(T X)=2 n+1-\delta$, see [27, Thm. 1.4]. Therefore $\operatorname{dim}(F)=\delta-1$. Reason as in the proof of Proposition 2.11 and observe that in our case $j=n+1-i$ and $i-j=2 i-(n+1)$. If $F$ is Fano, then $2 i-(n+1)=$ $i-j \leqslant \operatorname{dim}(F)+1=\delta$ with equality holding if and only if $F \simeq \mathbb{P}^{\delta-1}$, see [16]. Part (ii) is now clear.

(iii) The dual of the exact sequence (1) gives the map defining $X^{*}$, the dual of $X, \varphi^{\prime}: \mathbb{P}\left(N_{X / \mathbb{P}^{N}}(-1)\right) \rightarrow X^{*} \subset \mathbb{P}^{N^{*}}$; its Stein factorization is $\varphi: \mathbb{P}\left(N_{X / \mathbb{P}^{N}}(-1)\right) \rightarrow W, W$ being the normalization of $X^{*}$. Reason as above, note that $F$ is a linear space of dimension $k$ and that $j=n+1-i$. Since we have $i=\frac{n+k+2}{2}$, we get $i \geqslant \frac{n+3}{2}$ and $X$ is prime by Lemma2.3 (ii).

Remark 2.13. (i) For prime Fano manifolds of index $i$, the same condition $i \geqslant \frac{n+2}{2}$, as in Corollary 2.12 (i), ensures that $\mathcal{L}_{x} \subset \mathbb{P}^{n-1}$ is (non-empty and) non-degenerate, see [9. Thm. 2.5].

(ii) Corollary 2.12(iii) proves the Landman Parity Theorem for Fano manifolds: if $k>0, n$ and $k$ have the same parity. A variant of this argument proves the general case, when $X$ is only assumed to be dual defective, see [14, Prop. 3.1].

\section{FANO MANIFOLDS AND CC MANIFOLDS}

If $X \subset \mathbb{P}^{N}$ is $\mathrm{CC}$, then $X$ is Fano and classified unless it is prime of index $i$; if it is prime of index $i$, then $\delta>0$ and $i \geqslant \frac{n+1}{2}$, see [12] and [11, Prop. 3.2].

Is it true that, conversely, if $X \subset \mathbb{P}^{N}$ is a prime Fano manifold of index $i$, such that $i \geqslant \frac{n+1}{2}$ and $\delta>0$, then $X$ is $C C$ ?

The question is motivated by the following result of Bonavero-Höring, [4], which shows that the answer is affirmative in the "standard" case:

Proposition 3.1 ([4]). Let $X \subset \mathbb{P}^{N}$ be a complete intersection. Assume that $X$ is Fano of index $i \geqslant \frac{n+1}{2}$. Then $X$ is $C C$.

Lemma 3.2. Let $X \subset \mathbb{P}^{N}$ be a complete intersection. Assume that $X$ is Fano of index $i \geqslant \frac{n+r}{2}$, for some $r>0$. Then $\delta \geqslant r$.

Proof. Let $\left(d_{1}, \ldots, d_{c}\right)$ be the type of $X$. We have $i=n+1-\sum_{j=1}^{c}\left(d_{j}-1\right)$, which gives $2 c \leqslant 2 \sum_{j=1}^{c}\left(d_{j}-1\right) \leqslant n-r+2$. Finally, we get $\delta \geqslant$ $n-c+1 \geqslant r+c-1 \geqslant r$. 
Remark 3.3. (i) The above lemma shows that for a Fano complete intersection of index $i$, the condition $i \geqslant \frac{n+1}{2}$ implies that $\delta>0$.

(ii) The Lagrangian Grassmannian $\mathbb{L} \mathbb{G}(2,5) \subset \mathbb{P}^{13}$ is a prime Fano manifold of dimension 6 and index 4 (so we have $i=\frac{n+2}{2}$ ), but it has $\delta=0$, see the tables in [15].

At present the answer to the above question is not known and the only (very) partial results require stronger assumptions and seek for a stronger conclusion, namely that $X$ be connected by degenerate conics. In particular, we need to assume that $\delta \geqslant 2$.

Lemma 3.4. (i) If $X \subset \mathbb{P}^{N}$ is connected by degenerate conics, then $\delta \geqslant$ 2 ;

(ii) assume that $X$ is Fano of index $i$ and $C C$; then $i \geqslant \frac{n+2}{2}$ if and only if $X$ is connected by degenerate conics.

Proof. (i) Fix two general points $x, x^{\prime} \in X$. By assumption, there exists a degenerate conic $C_{x, x^{\prime}}=l \cup l^{\prime} \subset X$ with $x \in l$ and $x^{\prime} \in l^{\prime}$. Since $x$ and $x^{\prime}$ are general points, $N_{l / X}$ and $N_{l^{\prime} / X}$ are semiample and $C_{x, x^{\prime}}$ may be deformed inside $X$, by keeping the point $x$ fixed, to a smooth conic, say $C_{x}^{\prime}$, see [17, II.7.6.2]. Deformations of $C_{x, x^{\prime}}$ keeping $x$ fixed fill up $X$, so the same is true for the deformations of $C_{x}^{\prime}$. Therefore, through the general points $x, x^{\prime \prime} \in X$ there is a smooth conic $C_{x, x^{\prime \prime}}^{\prime}$. By hypothesis, there is also a degenerate conic $C_{x, x^{\prime \prime}}^{\prime \prime}$ through these points. The union $C_{x, x^{\prime \prime}}^{\prime} \cup C_{x, x^{\prime \prime}}^{\prime \prime}$ is part of the entry locus $\Sigma_{p}$ with respect to a general point $p$ of the line $\left\langle x, x^{\prime \prime}\right\rangle$. But $\Sigma_{p}$ is smooth at the points $x, x^{\prime \prime}$ and this shows that $\delta=\operatorname{dim}\left(\Sigma_{p}\right) \geqslant 2$.

(ii) This follows from [11, Prop. 3.2].

Proposition 3.5 ([[10]). Let $X \subset \mathbb{P}^{N}$ be Fano of index $i$, with $i \geqslant \frac{n+3}{2}$. Then the following are equivalent:

(i) $S \mathcal{L}_{x}=\mathbb{P}^{n-1}$ for a general point $x \in X$;

(ii) $X$ is connected by degenerate conics.

In the sequel we only need that (i) implies (ii), so we include a very simple proof of this result, based on the Terracini Lemma.

Proof. (i) implies (ii). We remark first that Lemma 2.3 implies that $X$ is prime Fano; moreover, by Lemma $2.5 X$ is covered by lines, so (i) makes sense. The condition $i \geqslant \frac{n+3}{2}$ is equivalent to $a \geqslant \frac{n-1}{2}$; therefore $\mathcal{L}_{x} \subset$ $\mathbb{P}^{n-1}$ is smooth, irreducible and non-degenerate, for $x \in X$ a general point, see [9]. Let $l \subset X$ be a general line and let $x, x^{\prime} \in l$ be general points. Let $\left[l^{\prime}\right] \in \mathcal{L}_{x^{\prime}}$ be a general line and let $e \in l^{\prime}$ be a general point. Denote by $C(x)$ the locus of lines through $x \in X$; it is a cone of dimension $a+1$. 
By the Terracini Lemma, the condition $S \mathcal{L}_{x}=\mathbb{P}^{n-1}$ is equivalent to (2)

$$
\operatorname{dim}\left(T_{x} C\left(x^{\prime}\right) \cap T_{e} C\left(x^{\prime}\right)\right)=2 a+2-n .
$$

Denote by $\mathrm{E}$ the locus of points lying on lines meeting $C(x) . X$ is connected by degenerate conics exactly when $E=X$; equivalently, $\operatorname{dim}(E) \geqslant n$.

If $\pi: \mathcal{Y} \rightarrow \mathcal{L}$ is the family of lines covering $X$ and $\psi: \mathcal{Y} \rightarrow X$ is the projection, let $V:=\pi^{-1}\left(\pi\left(\psi^{-1}(C(x))\right)\right)$. Note that $\operatorname{dim}(V)=2 a+2$. $\operatorname{dim}(E) \geqslant n$ is the same as asking that, for some $e \in V$, the fiber $V_{e}$ of the map $\left.\psi\right|_{V}: V \rightarrow E$ satisfies $\operatorname{dim}\left(V_{e}\right) \leqslant 2 a+2-n$. But $\operatorname{dim}\left(V_{e}\right)=$ $\operatorname{dim}_{x^{\prime}}(C(x) \cap C(e))$.

Therefore, keeping the above notation, to prove that $X$ is connected by degenerate conics, we have to show that

$$
\operatorname{dim}_{x^{\prime}}(C(x) \cap C(e)) \leqslant 2 a+2-n .
$$

Let us recall from [14, Prop. 2.2] that, if $x, x^{\prime}$ are general points of a general line $l \subset X$, we have

$$
T_{x} C\left(x^{\prime}\right)=\bigcap_{y \in l} T_{y} X=T_{x^{\prime}} C(x) .
$$

Now, using (2) and (4), we can write

$$
\begin{aligned}
\operatorname{dim}_{x^{\prime}}(C(x) \cap C(e)) & \leqslant \operatorname{dim}\left(T_{x^{\prime}} C(x) \cap T_{x^{\prime}} C(e)\right) \\
& =\operatorname{dim}\left(T_{x} C\left(x^{\prime}\right) \cap T_{e} C\left(x^{\prime}\right)\right)=2 a+2-n
\end{aligned}
$$

and (3) is proved.

Note that, since $X$ is smooth, the inequality $\operatorname{dim}_{x^{\prime}}(C(x) \cap C(e)) \geqslant 2 a+$ $2-n$ also holds; therefore, the cones $C(x)$ and $C(e)$ intersect transversally at $x^{\prime}$.

Corollary 3.6 ([10], see also [12]). Let $X \subset \mathbb{P}^{N}$ be Fano of index $i$ and assume that $i>\frac{2 n}{3}$. Then $X$ is connected by degenerate conics.

Remark 3.3 (ii) shows that the bound $i>\frac{2 n}{3}$ is optimal.

Proposition 3.5 implies Proposition 3.1 in case $i \geqslant \frac{n+2}{2}$ :

Proposition 3.7. Let $X \subset \mathbb{P}^{N}$ be a complete intersection and assume $X$ is Fano, of index $i$. The following are equivalent:

(i) $i \geqslant \frac{n+2}{2}$;

(ii) $X$ is connected by degenerate conics.

Proof. (i) implies (ii):

The result is clear for $n \leqslant 2$, so we may assume $n \geqslant 3$ from now on. It follows from (i) that, for $x \in X$ a general point, $\mathcal{L}_{x} \subset \mathbb{P}^{n-1}$ is a smooth, non-degenerate, complete intersection of positive dimension $a=i-2$. In 
particular, it is connected and hence irreducible. Moreover, since it is a complete intersection, it follows that $S \mathcal{L}_{x}=\mathbb{P}^{n-1}$, see Lemma 2.7. Then (ii) follows by the proof of Proposition 3.5

(ii) implies (i) follows from Lemma 3.4

Proposition 3.8. Let $X \subset \mathbb{P}^{N}$ be Fano of index $i$ and suppose $\delta \geqslant 2$. Assume that $i>\frac{n+1}{2}+\frac{\delta}{4}$. Then $X$ is connected by degenerate conics.

Proof. Note that Lemma 2.3 implies that $X$ is prime Fano. Let $Y=\mathbb{P}\left(\mathcal{P}_{X}\right)$ and let $\varphi: Y \rightarrow W$ be as in the proof of Corollary 2.12 By Corollary 2.12 (i), the general fiber $F$ of $\varphi$ is Fano and $i(F)=2 i-n-1>$ $\frac{\operatorname{dim}(F)+1}{2}=\frac{\delta}{2}$. This implies that $F$ is covered by lines, see Lemma 2.5. But $F$ passes through the general point of $Y$; so $Y$ is covered by a family of lines, projecting onto lines of $X$ and contracted by the map $\varphi^{\prime}: Y \rightarrow T X$. We claim that this implies $S \mathcal{L}_{x}=\mathbb{P}^{n-1}$. By Proposition 3.5, it follows that $X$ is conic-connected (by degenerate conics).

Fix a point $y \in Y$ and let $x=\pi(y)$. Assume that a line $l \subset Y, y \in l$ is contracted to the general point $u \in T X$. It follows that all (projective) tangent spaces to $X$ at points of $\pi(l)$ pass through the point $u$. But, for $s \in l$ a general point, the intersection $\bigcap_{t \in l} T_{t} X$ is the projective tangent space to the cone of lines through $s$, at a general point of $l$, see (4). As the point $u \in T_{x} X$ is general, this means that $T \mathcal{L}_{x}=\mathbb{P}^{n-1}$, so we have a fortiori that $S \mathcal{L}_{x}=\mathbb{P}^{n-1}$.

Corollary 3.9. Let $X \subset \mathbb{P}^{N}$ be Fano of index $i$ with $\delta \geqslant 2$. Then we have:

(i) $i \leqslant \frac{n+\delta}{2}$; in particular, if $i \geqslant \frac{n+r}{2}$ for some $r>0$, then $\delta \geqslant r$ (cf. Lemma 3.2). If $S X=\mathbb{P}^{N}$, this becomes $c \leqslant n-r+1$.

(ii) The general fiber of the map $\varphi: \mathbb{P}\left(\mathcal{P}_{X}\right) \rightarrow W$ is not linear.

Proof. (i) Suppose that $i>\frac{n+\delta}{2}$; since $\delta \geqslant 2$, we get $i>\frac{n+1}{2}+\frac{\delta}{4}$. By Proposition 3.8, $X$ is conic-connected. It follows that $i \leqslant \frac{n+\delta}{2}$, by [11, Prop. 3.2].

(ii) It follows from the previous inequality and part (i) of Corollary 1.

\section{FANO MANIFOLDS AND LQEL MANIFOLDS}

Proposition 4.1. Assume that $X \subset \mathbb{P}^{N}$ is Fano of index $i$ and $\delta \geqslant 3$. The following conditions are equivalent:

(i) $X$ is $L Q E L$;

(ii) $i=\frac{n+\delta}{2}$; 
(iii) the general fiber of the map $\varphi: \mathbb{P}\left(\mathcal{P}_{X}\right) \rightarrow W$ is isomorphic to the quadric $Q^{\delta-1}$;

(iv) if $x \in X$ is a general point, we have $S \mathcal{L}_{x}=\mathbb{P}^{n-1}$ and $\delta(X) \leqslant$ $\delta\left(\mathcal{L}_{x}\right)+2$.

Proof. Since $\delta \geqslant 3, X$ is prime Fano, see [2]. (i) implies (ii) and (i) implies (iv) follow from [24]. If $F$ is a general fiber of $\varphi$, the equivalence of (ii) and (iii) comes from the formula in Proposition 2.11 $i(F)=2 i(X)-n-1$, and the fact that $Q^{r}$ is characterized by having its index equal to $r$, see [16]. (ii) implies (i) comes from Proposition 3.8 and [11, Prop. 3.2]. Finally, from (iv) we infer $\delta(X) \leqslant \delta\left(\mathcal{L}_{x}\right)+2=a-(n-1-a)+1+2=2 a+4-n=2 i-n$, so (ii) holds by using also Corollary 3.9

The next result shows that Fano manifolds of high index and small codimension are very special.

Proposition 4.2. Let $X \subset \mathbb{P}^{N}$ be Fano of index $i$. Assume that $i \geqslant n-\frac{c-1}{2}$ and $n \geqslant 2 c-2$. Then $X$ is an LQEL manifold and one of the following holds (up to an automorphism of $\mathbb{P}^{N}$ ):

(i) $c=1, n \geqslant 1$ and $X$ is $Q^{n} \subset \mathbb{P}^{n+1}$;

(ii) $n=2 c$ and $X$ is one of: $\mathbb{G}(1,4) \subset \mathbb{P}^{9}$ or $S^{10} \subset \mathbb{P}^{15}$, in their natural embeddings;

(iii) $n=2 c-1$ and $X$ is one of: $\mathbb{G}(1,4) \cap H \subset \mathbb{P}^{8}$ or $S^{10} \cap H \subset \mathbb{P}^{14}$, where $H, H^{\prime}$ are general hyperplanes in $\mathbb{P}^{N}$;

(iv) $n=2 c-2$ and $X$ is one of: $\mathbb{G}(1,4) \cap H \cap H^{\prime} \subset \mathbb{P}^{7}, S^{10} \cap H \cap H^{\prime} \subset \mathbb{P}^{13}$, or one of the following Severi varieties:

$\mathbb{P}^{2} \times \mathbb{P}^{2} \subset \mathbb{P}^{7}$, projection of the Segre embedding into $\mathbb{P}^{8}$, $\mathbb{G}(1,5) \subset \mathbb{P}^{13}$, projection of the Plücker embedding into $\mathbb{P}^{14}$ and $E_{6} \subset \mathbb{P}^{25}$, projection of the natural embedding into $\mathbb{P}^{26}$.

Proof. Since $n \geqslant 2 c-2$, it follows from Zak's Linear Normality Theorem, see [27], that we have $S X=\mathbb{P}^{N}$. Assume first that $c \geqslant 4$; we get $\delta=$ $n-c+1 \geqslant c-1 \geqslant 3$. The hypothesis $i \geqslant n-\frac{c-1}{2}$ reads $i \geqslant \frac{n+\delta}{2}$ and from Corollary 3.9 and Proposition 4.1 it follows that $X$ is an LQEL manifold. Now the classification follows from the results in section 3 of [24]; due to [18], the last case of [24, Cor. 3.2] is ruled out.

Assume next that $c \leqslant 3$. For $c=1$ we get case (i) by [16]. The case $c=2$ is impossible. Finally, suppose that $c=3$. We infer that $X$ is a del Pezzo manifold, see [8] for their classification. From the condition $c=3$ we deduce $n \geqslant 4$ and we get the Grassmannian $\mathbb{G}(1,4) \subset \mathbb{P}^{9}$ and its linear sections that are mentioned in the cases (ii), (iii) and (iv). 
Proposition 4.3. Let $X \subset \mathbb{P}^{N}$ be an LQEL manifold and assume that $\delta \geqslant 3$. Let $Q$ be a general fiber of $\varphi: \mathbb{P}\left(\mathcal{P}_{X}\right) \rightarrow W$. Then we have:

(i) $N_{Q / X}$ is spanned;

(ii) $\left.\mathcal{P}_{X}\right|_{Q}$ is spanned, uniform, with splitting type $(0, \ldots, 0,1, \ldots, 1)$ and degree $n-1-a$ on any line contained in the quadric $Q$.

Proof. (i) The standard diagram

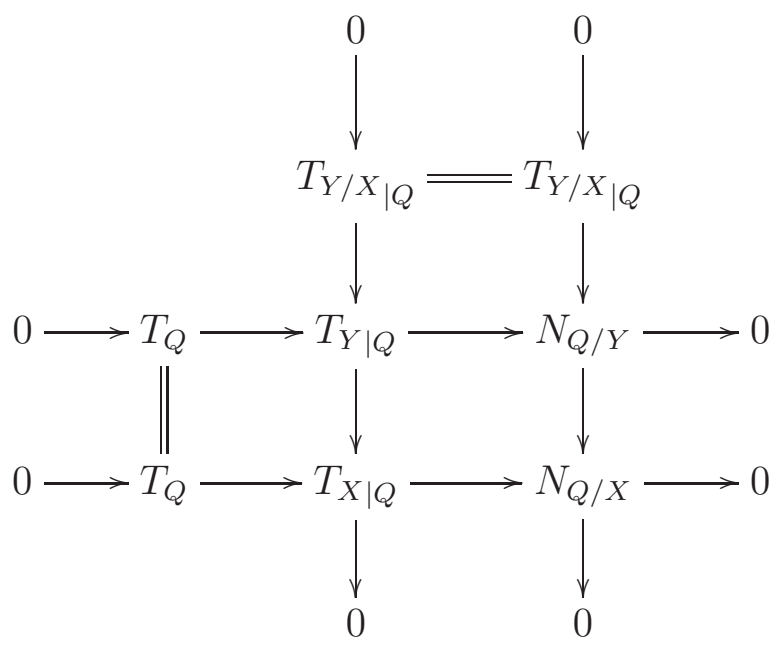

shows that $N_{Q / X}$ is a quotient of the trivial vector bundle $N_{Q / Y}$, where $Y=\mathbb{P}\left(\mathcal{P}_{X}\right)$, see Corollary 2.12(i).

(ii) If $l \subset Q$ is a line, from the standard exact sequence

$$
\left.0 \longrightarrow N_{l / Q} \longrightarrow N_{l / X} \longrightarrow N_{Q / X}\right|_{l} \longrightarrow 0
$$

it follows that $N_{l / X}$ is spanned. Therefore its splitting type is $(0, \ldots, 0,1, \ldots, 1)$ and the conclusion about $\left.\mathcal{P}_{X}\right|_{l}$ follows from [14, Prop. 2.2].

\section{FANO MANIFOLDS AND DD MANIFOLDS}

In this section we collect a number of facts that support our belief that any DD manifold which is Fano of index $i$ is LQEL.

Remark 5.1. (i) If $X \subset \mathbb{P}^{N}$ is DD, it follows from Zak's Theorem on Tangencies, [27], that we have $k \leqslant c-1$.

(ii) If $X \subset \mathbb{P}^{N}$ is LQEL and $X \neq Q^{n}$, we have $\delta \leqslant c+1$, see [14, Prop. 4.2].

Proposition 5.2. Let $X \subset \mathbb{P}^{N}$ be Fano. Assume that $X$ is DD. Then $X$ is $L Q E L$ if and only if $\delta=k+2$. 
Proof. Since $X$ is DD, we have $i=\frac{n+k+2}{2}$; since $X$ is LQEL, we have $i=\frac{n+\delta}{2}$. So, $\delta=k+2$. Conversely, if $k=\delta-2$, it follows that $i=\frac{n+\delta}{2}$ and $X$ is LQEL by Proposition 4.1.

Proposition 5.3. Let $X \subset \mathbb{P}^{N}$ be a DD manifold which is Fano of index $i$.

Then we have:

(i) If $\delta \geqslant 2$, then $\delta \geqslant k+2$; if $\delta=3$, then $X$ is $L Q E L$;

(ii) assume that $k \geqslant 2$. Then one of the following holds:

(a) $\delta \geqslant k+2$, or

(b) $n \leqslant 2 c-1$ and for $x \in X$ general, $\mathcal{L}_{x} \subset \mathbb{P}^{n-1}$ is prime Fano and covered by lines;

(iii) $c f$. [11] if $k>\frac{n-6}{3}$, then $X$ is $C C$ and $\delta \geqslant k+2$;

(iv) [14] we have that $k \leqslant \frac{n+2}{3}$ and equality holds exactly when $X$ is $S^{10} \subset \mathbb{P}^{15}$;

(v) if $k \geqslant \min \left\{\frac{n-4}{2}, \frac{n+2}{3}\right\}$, then $X$ is LQEL;

(vi) cf. [5] if $k=c-1$ and $n \leqslant \max \{2 c+2,3 c-5\}$, then $X$ is one of $\mathbb{G}(1,4) \subset \mathbb{P}^{9}$ or $S^{10} \subset \mathbb{P}^{15}$

(vii) $c f$. [21] if $k=c-2$ and $n \leqslant 2 c$, then $X \subset \mathbb{P}^{N}$ is a hyperplane section of one of the two varieties in (vi).

Assume now that $X$ is LQEL and $X \neq Q^{n}$; then we have:

(viii) If $\delta=c+1$, then $X \subset \mathbb{P}^{N}$ is one of the two varieties in (vi);

(ix) $c f$. [7] $\delta \leqslant \frac{n+8}{3}$ and equality holds exactly when $X$ is one of $S^{10} \subset \mathbb{P}^{15}$, $E_{6} \subset \mathbb{P}^{26}$, or its isomorphic projection to $\mathbb{P}^{25}$.

Proof. By Corollary 2.12(iii), $X$ is prime.

(i) The first part follows from Corollary 3.9 (i), given that $i=\frac{n+k+2}{2}$. If $\delta=3$, we get $3=\delta \geqslant k+2$, so that $k=1=\delta-2$ and Proposition 5.2 applies.

(ii) Assume first that $n \geqslant 2 c$. It follows that $\delta \geqslant n-c+1 \geqslant 2$. Therefore $\delta \geqslant k+2$, by (i). Assume from now on that $n \leqslant 2 c-1$. As we have $i \geqslant \frac{n+3}{2}$, we know that $\mathcal{L}_{x} \subset \mathbb{P}^{n-1}$ is smooth, non-degenerate and irreducible, see [9]. If $S \mathcal{L}_{x}=\mathbb{P}^{n-1}$, by Proposition 3.5 $X$ is connected by degenerate conics, so $\delta \geqslant 2$, see Lemma 3.4 (i). So we again get that $\delta \geqslant k+2$ by (i). Now we may assume that $S \mathcal{L}_{x} \neq \mathbb{P}^{n-1}$ and hence $\delta\left(\mathcal{L}_{x}\right)>a-(n-1-a)+1=k \geqslant 2$. By the Barth-Larsen Theorem, [2], we get that $\operatorname{Pic}\left(\mathcal{L}_{x}\right)$ is cyclic, generated by the hyperplane section class. On the other hand, we have $n-2 c+2 \leqslant 1 \leqslant k$ and it follows from [14, Prop. 3.2] that all lines in $X$ are contact lines. Therefore $\mathcal{L}_{x}$ is covered by lines. As its Picard group is cyclic, generated by the hyperplane section, it is prime Fano, of some index $j$. 
(iii) Since we have $2 i=n+k+2$, the condition $k>\frac{n-6}{3}$ is equivalent to $i>\frac{2 n}{3}$. So $X$ is connected by degenerate conics by Corollary 3.6 . From Lemma 3.4, we have $\delta \geqslant 2$, so (i) applies.

(iv) See [14, Cor. 3.5].

(v) Using (iv) and [6], we are left with the cases $n \leqslant 15$ and $n=2 k+4$ or $n=2 k+3$. When $n \leqslant 10$ we may use the classification in [3], together with [8] and [20]. The only remaining case is $n=12, k=4, i=9$. By Zak's Theorem on Tangencies we have $k \leqslant c-1$, so that $c \geqslant 5$. Therefore, $4=k \geqslant n-2 c+2$, so that all lines on $X$ are contact, see [14, Prop. 3.2]. Let $x \in X$ be a general point and $X^{\prime}:=\mathcal{L}_{x} \subset \mathbb{P}^{11}$. We have $\operatorname{dim}\left(X^{\prime}\right)=i-2=7$ and $\operatorname{codim}\left(X^{\prime}\right)=4$, so $X^{\prime} \subset \mathbb{P}^{11}$ is prime Fano and covered by linear spaces of dimension three. Let $x^{\prime} \in X^{\prime}$ be a general point and let $X^{\prime \prime}:=\mathcal{L}_{x^{\prime}} \subset \mathbb{P}^{6}$. If $\operatorname{dim}\left(X^{\prime \prime}\right) \geqslant 3$, we have $i\left(X^{\prime}\right)=\operatorname{dim}\left(X^{\prime \prime}\right)+2 \geqslant 3+2=5$. So, $X^{\prime}$ would be a del Pezzo or Mukai prime Fano manifold, which is excluded by their classification, see [8] and [20]. Since $X^{\prime}$ is covered by linear spaces of dimension three, we are left with the case where $\operatorname{dim}\left(X^{\prime \prime}\right)=2$. The following argument has been suggested by the referee. We let $L \subset X^{\prime}$ be a linear space (of dimension three) passing through the general point $x^{\prime} \in X^{\prime}$ and we let $x^{\prime} \in l \subset L$ be a line. From the standard exact sequence

$$
\left.0 \longrightarrow N_{l / L} \longrightarrow N_{l / X^{\prime}} \longrightarrow N_{L / X^{\prime}}\right|_{l} \longrightarrow 0
$$

it follows that $\left.N_{L / X^{\prime}}\right|_{l}$ is nef and its degree is zero, so it is trivial. Applying [23, Thm. 3.2.1], we get that $N_{L / X^{\prime}}$ is trivial. Now we may quote [22, Prop. $6.4]$ to exclude this last possibility.

(vi) If $n \leqslant 3 c-5$, we get $k \geqslant \frac{n+2}{3}$ and (iv) applies. If $n \leqslant 2 c+2$, we have $k \geqslant \frac{n-4}{2}$ and it follows from (v) that $X$ is LQEL. But an LQEL manifold satisfies the condition $\delta=k+2=c+1$. From Lemma 5.4 below, the condition $\delta=c+1$ is equivalent to $n=2 c$. The result follows now from [24, Cor. 3.1].

(vii) As above, it follows from (iv) and (v) that $X$ is LQEL. Therefore we have $\delta=k+2=c$, so $c=\delta \geqslant n-c+1$ and $n \leqslant 2 c-1$. This gives $\delta=c>\frac{n}{2}$ and the result again follows from [24, Cor. 3.1].

(viii) From Lemma 5.4 below, $\delta=c+1$ is equivalent to $n=2 c$, so the result follows from [24, Cor. 3.1].

(ix) We may assume that $\delta \geqslant 5$. We know that $X^{\prime}:=\mathcal{L}_{x} \subset \mathbb{P}^{n-1}$ is also an LQEL, having dimension $a=\frac{n+\delta-4}{2}$ and secant defect $\delta^{\prime}=\delta-2$, see [24, Thm. 2.8]. The bound $\delta \leqslant \frac{n+8}{3}$ follows quickly from this, see [14, Cor. 4.4]. If equality holds, we get that $\delta^{\prime}>\frac{a}{2}$ and from [24, Cor. 3.1] we deduce that $X^{\prime}$ is one of $\mathbb{G}(1,4) \subset \mathbb{P}^{9}$ or $S^{10} \subset \mathbb{P}^{15}$. Using [18], we get the three cases announced in (ix). 
Lemma 5.4. Let $X \subset \mathbb{P}^{N}$ be smooth, non-degenerate. The following conditions are equivalent:

(i) $n \leqslant 2 c$;

(ii) $\delta \leqslant c+1$.

Moreover, equality holds in (i) if and only if it holds in (ii).

Proof. (ii) implies (i): We have $c+1 \geqslant \delta \geqslant n-c+1$, so $n \leqslant 2 c$.

(i) implies (ii): If $S X=\mathbb{P}^{N}$, we have $\delta=n-c+1$ and (ii) is clear. Otherwise, by Zak's Linear Normality Theorem, see [27], we have $\delta \leqslant \frac{n}{2}$, so $\delta \leqslant c$.

The other assertion of the lemma is proved in a similar way.

Proposition 5.5. Assume that $X$ is Fano and DD. Let $\mathbb{P}^{k} \cong L \subset X$ be the contact locus of a general hyperplane. Then we have:

(i) $N_{L / X}$ is spanned;

(ii) $\left.\mathcal{P}_{X}\right|_{L}$ is spanned, uniform, with splitting type $(0, \ldots, 0,1, \ldots, 1)$ and degree $n-1-a$ on any line contained in $L$.

Proof. We proceed as in the proof of Proposition 4.3, Let $Y:=\mathbb{P}\left(N_{X / \mathbb{P}^{N}}(-1)\right)$ and note that $L$ is the general fiber of the morphism $\varphi: \mathbb{P}\left(N_{X / \mathbb{P}^{N}}(-1)\right) \rightarrow$ $W$, see Corollary 2.12 (iii). Therefore the normal bundle $N_{L / Y}$ is trivial and a diagram similar to the one from the proof of Proposition 4.3 shows that $N_{L / X}$ is spanned. Now, if $l \subset L$ is a line, the exact sequence:

$$
\left.0 \longrightarrow N_{l / L} \longrightarrow N_{l / X} \longrightarrow N_{L / X}\right|_{l} \longrightarrow 0
$$

gives that $N_{l / X}$ is spanned and the conclusions follow from [14, Prop. 2.2].

\section{REMARKS ON THE QUADRATIC CASE AND THE HARTSHORNE CONJECTURE}

Definition 6.1. $X \subset \mathbb{P}^{N}$ is quadratic if it is scheme-theoretically an intersection of quadrics.

Remark 6.2. (i) All known examples of linearly normal LQEL or DD prime Fano manifolds are quadratic.

(ii) All known examples of prime Fano manifolds of high index, in their natural linearly normal embedding, are either complete intersections or quadratic.

(iii) Quadratic manifolds of small codimension $(n \geqslant c+1)$ are quite restricted: they are Fano, covered by lines and $\mathcal{L}_{x} \subset \mathbb{P}^{n-1}$ is also quadratic, see [13]. 
(iv) If $X \subset \mathbb{P}^{N}$ is quadratic and covered by lines, the splitting-type of $N_{X / \mathbb{P}^{N}}(-1) \mid l$ is $(0, \ldots, 0,1, \ldots, 1)$ if $l$ is a general line. We recall from [14. Prop. 3.2] that, if $X$ is DD and $k \geqslant n-2 c+2$, this property still holds.

Let us also recall the famous Hartshorne Conjecture, HC for short:

If $n \geqslant 2 c+1, X$ is a complete intersection.

We consider it very plausible for (prime) Fano manifolds. The HC holds in the following special cases: for Fano manifolds in codimension two [1], for quadratic manifolds [13], for LQEL manifolds and for $\mathcal{L}_{x} \subset \mathbb{P}^{n-1}$ if $X$ is $\mathrm{DD}$, see [14].

Both LQEL and DD Fano manifolds have high index and we expect the $\mathrm{HC}$ to be easier to prove in this case. An example is Proposition 4.2, where "high index" means $i \geqslant n-\frac{c-1}{2}$. This inequality excludes all complete intersections, but for the hyperquadrics. We close this note with another partial result of the same flavor, where only complete intersections should be present.

Proposition 6.3. Let $X \subset \mathbb{P}^{N}$ be Fano of index $i \geqslant \frac{N+2}{2}$. Then the following hold:

(i) $X$ is prime and covered by lines;

(ii) $X$ is connected by degenerate conics;

(iii) $S X=\mathbb{P}^{N}$;

(iv) $n \geqslant 2 c+1$.

Proof. (i) The fact that $X$ is prime follows from Lemma 2.3, Lemma 2.5 shows that $X$ is covered by lines.

(ii) If $x, x^{\prime} \in X$ are general points, let $C(x), C\left(x^{\prime}\right)$ be the cones of lines passing through the respective points and contained in $X$; they have dimension $a+1$. Since we have $i=a+2$, it follows that $2 a+2 \geqslant N$, so the cones $C(x)$ and $C\left(x^{\prime}\right)$ must intersect.

(iii) and (iv) From (ii) and Lemma 3.4 it follows that $\delta \geqslant 2$. Now, Corollary 3.9 yields that $\delta \geqslant c+2$. By Lemma 5.4 we get that $n \geqslant 2 c+1$ and the conclusion follows from Zak's Linear Normality Theorem.

Properties (i), (ii) and (iii) are necessary conditions for $X$ to be a complete intersection and, by (iv), the $\mathrm{HC}$ predicts this is indeed the case. Note that the inequality $i \geqslant \frac{N+2}{2}$ is optimal, as the examples $\mathbb{G}(1,4) \subset \mathbb{P}^{9}$ and $S^{10} \subset \mathbb{P}^{15}$ show.

\section{REFERENCES}


W. BARTh, M.E. LARSEn, On the homotopy groups of complex projective algebraic manifolds, Math. Scand. 30 (1972), 88-94. M.C. Beltrametti, M.L. Fania, A.J. Sommese, On the discriminant variety of a projective manifold, Forum Math. 4 (1992), 529-547.

L. BONAVERO, A. HöRING, Counting conics in complete intersections, Acta Math. Vietnamica 35 (2010), 23-30.

L. EIN, Varieties with small dual variety. I, Invent. Math. 86 (1986), 63-74. L. EIN, Varieties with small dual variety. II, Duke Math. J. 52 (1985), 895907.

B. FU, Inductive characterizations of hyperquadrics, Math. Ann. 340 (2008), 185-194.

T. Fujita, Classification Theories of Polarized Varieties, London Math. Soc. Lecture Note Ser., vol. 155, Cambridge Univ. Press, 1990.

J.M. HWANG, Geometry of minimal rational curves on Fano manifolds, in School on Vanishing Theorems and Effective Results in Algebraic Geometry (Trieste, 2000), ICTP Lect. Notes, vol. 6, Abdus Salam Int. Cent. Theoret. Phys., 2001, pp. 335-393.

J.M. HWANG, S. KeBEKUS, Geometry of chains of minimal rational curves, J. Reine Angew. Math. 584 (2005), 173-194.

P. IONESCU, F. Russo, Varieties with quadratic entry locus. II, Compositio Math. 144 (2008), 949-962.

P. Ionescu, F. Russo, Conic-connected manifolds, J. Reine Angew. Math. 644 (2010), 145-157.

P. IONESCU, F. Russo, Manifolds covered by lines and the Hartshorne Conjecture for quadratic manifolds, Amer. J. Math. 135 (2013), 349-360.

P. Ionescu, F. Russo, On dual defective manifolds, Math. Res. Lett. 21 (2014), 1137-1154.

H. KAJI, Homogeneous projective varieties with degenerate secants, Trans. Amer. Math. Soc. 351 (1999), 533-545.

S. Kobayashi, T. OChIAI, Characterizations of complex projective spaces and hyperquadrics, J. Math. Kyoto Univ. 13 (1973), 31-47.

J. Kollár, Rational Curves on Algebraic Varieties, Ergeb. Math. Grenzgeb. (3), vol. 32, Springer, 1996.

N. MoK, Recognizing certain rational homogeneous manifolds of Picard number 1 from their varieties of minimal rational tangents, in Third International Congress of Chinese Mathematicians. Part 1, 2. AMS/IP Studies in Advanced Mathematics, vol. 42, pt. 1, 2 (American Mathematical Society, Providence, RI, 2008), pp. 41-61.

S. MORI, Projective manifolds with ample tangent bundle, Ann. of Math. 110 (1979), 593-606.

S. MUKAI, Biregular classification of Fano 3-folds and Fano manifolds of coindex 3, Proc. Nat. Acad. Sci. USA 86 (1989), 3000-3002.

R. MUÑOZ, Varieties with low dimensional dual variety, Manuscripta Math. 94 (1997), 427-435.

C. Novelli, G. Occhetta, Projective manifolds containing a large linear subspace with nef normal bundle, Michigan Math. J. 60 (2011), 441-462.

C. OKoneK, M. Schneider, H. Spindler, Vector Bundles on Complex Projective Spaces, Progress in Mathematics 3, Springer, 1980. 
[24] F. Russo, Varieties with quadratic entry locus. I, Math. Ann. 344 (2009), 597-617.

[25] F. Russo, On the Geometry of Some Special Projective Varieties, Lecture Notes of the Unione Matematica Italiana, Springer, 2016.

[26] J. WIŚNIEWSKI, On a conjecture of Mukai, Manuscripta Math. 68 (1990), $135-141$.

[27] F.L. ZAK, Tangents and Secants of Algebraic Varieties, Transl. Math. Monogr., vol. 127, Amer. Math. Soc., 1993.

P. IONESCU, Dipartimento di MATEMATICA E INFORMATICA, UniVERSitÀ DEGLi StUdi di Ferrara, Via Machiavelli, 30, 44121 Ferrara, Italy,

EMAIL: PALTIN.IONESCUUNIFE.IT

F. Russo, Dipartimento di Matematica e Informatica, Università degli Studi di Catania, Viale A. Doria, 6, 95125 Catania, Italy

EMAIL: FRUSSODMI.UNICT.IT 